\title{
Culturally competent physician: A need of the day
}

\author{
Chandanie Wanigatunge ${ }^{1}$ \\ Journal of the Ceylon College of Physicians, 2021, 52, 1-3
}

\begin{abstract}
"Science and medicine must be practiced in the context of human desires and needs. To provide holistic care, a physician must understand the human being, his society and environment."
\end{abstract}

Galen (c. 129 - c. 216/17 CE)

Culture is a pattern of learned beliefs, values, and behaviour that are shared within a group and includes language, styles of communication, practices, customs, and views on roles and relationships. ${ }^{1}$ Each culture has its own core beliefs and practices and are influenced by societal norms, religious beliefs, personal experiences and the practices related to traditional medicine which in turn influence health and medical practices. ${ }^{2}$ Healthcare services in any country are accessed by culturally and linguistically diverse patients whose beliefs influence healthcare seeking behaviours and adherence to its practices. Beliefs of cautiousness results in patients being extra careful beyond medical advice (e.g., avoidance of head baths during illnesses) while beliefs of contradiction may result in patients not complying with instruction (e.g., reluctance to use inhalers in the belief that use of inhalers makes one dependent on them). In a system where alternative practices play a significant role, belief of alternatives could lead to patients having no faith in medical advice from an allopathic system and look for alternatives (e.g., those with epilepsy/ mental illnesses trying exorcism in the belief that these are illnesses due to influence of supernatural beings or going behind faith healers for cancers or incurable diseases). Being culturally competent would help healthcare providers to give better advice to patients and ensure greater compliance to such advice while reducing the disparities in accessing healthcare.

Cultural competence is defined as the ability of providers and organizations to effectively deliver healthcare services that meet the social, cultural, and linguistic needs of patients. ${ }^{3}$ The goal of cultural competence in healthcare is to reduce health disparities and to provide optimal care to patients regardless of their race, gender, ethnic background, native languages spoken, and religious or cultural beliefs. A culturally competent health system recognizes and accepts the importance of cultural diversity at every level, assesses the cross-cultural relations, stays vigilant towards any changes and developments resulting from cultural diversity, broadens cultural knowledge, and adapts services to meet the needs that are culturally unique. ${ }^{4}$ To provide culturally sensitive patient-centred care, physicians should treat each patient as an individual, recognizing and respecting his or her beliefs, values and care seeking behaviours. ${ }^{5}$ Failure to do so could lead to mistrust, dissatisfaction, decreased adherence resulting in poorer health outcomes. ${ }^{3}$

The concept of cultural competence gained importance with the recognition of poorer health status and disparities in health care delivery to ethnic minorities and migrants in developed countries. Despite the absence of migrants, being culturally competent is also important for developing countries with their multiethnic and multi religious communities and traditional medical systems that exist parallel to the allopathic system. The traditional systems in these countries play an important role in healthcare delivery and influence health seeking behaviour of the people. In a suburban Sri Lankan population, ayurvedic health care was the preferred initial treatment for most following fractures. ${ }^{6}$ The influence of traditional systems was also amply demonstrated during the COVID pandemic where people were extremely receptive to advice and treatment modalities originating from these systems despite lack of evidence for efficacy or safety.

${ }^{1}$ Professor of Pharmacology, Faculty of Medical Sciences, University of Sri Jayewardanepura, Sri Lanka.

Correspondence: E-mail: caw@sjp.ac.lk

https://orcid.org/0000-0002-4477-8979

This is an open-access article distributed under the terms of the Creative Commons Attribution License, which permits unrestricted use, distribution, and reproduction in any medium, provided the original author and source are credited. 
Physicians in such countries would belong to cultures at variance with the values of the allopathic system they have learnt and practice which could lead to conflicts. When faced with culture-related concepts, practitioners of the allopathic system may consider them as unimportant or unscientific and ignore the patient's beliefs if it doesn't fit with the bio-medical model. Alternatively, these beliefs may be entertained based on a culturally biased thinking. However, neither will achieve the desired outcomes.

The modern "Western" medical system originated in the West and has values and norms which may at times be different to those in countries that practice the system. For example, the Western concept of autonomy where the individual takes his/her own decisions is not perceived in the same manner in other countries where the family plays a significant role in decision making. This is widely encountered in caring for patients with malignancies where the family is against divulging the true nature of the illness to the patients. ${ }^{1}$ The physician then faces a dilemma as his learnings and practices are different to that of his patients' expectations and values. Understanding and working with such issues will provide an ethically accepted compromise to inform the patient in a manner acceptable to the family.

Being able to communicate in the patient's own language is important for effective communication. Patients may present their symptoms differently from how we hear them or learnt from textbooks. ${ }^{1}$ Words may have regional nuances - for example a single Sinhala word (ळூి心) is used by patients to describe shortness of breathing, wheezing or even chest tightness and if not properly understood and explored by the physician can lead to a wrong diagnosis and management. The issues with language can be made worse if the Physician is practicing in a region where the primary language is not his own. Using interpreters, in many instances whoever is around that speaks the patient's native language, results in information being "lost in translation" between the patient and his healthcare provider. While language competence is important, cultural competence is not merely the language skills needed to address language barriers. ${ }^{1}$ When faced with issues that are culturally diverse, physicians need to explore and reflect upon these issues from the patient's point of view. It is equally important to express the physicians' own views, negotiate reality and advocate appropriately. Such mutual understanding of issues will help the patient to be a partner in decision making and illness management.

While healthcare professionals need to learn about the tolerance of other's beliefs, it would be reasonable to encourage patients as well to be culturally sensitive and be aware that not all health care providers are equally competent in diverse cultures. Patients also should try their best to communicate their concerns relating to their beliefs, values and other cultural factors that might affect care and treatment to their physicians and other healthcare providers. A bidirectional dialogue without preconceived ideas or prejudices is essential to improve outcomes in clinical encounters.

Cultural competence can be taught and implemented during clinical training and will provide physicians with the knowledge and skills needed to address cross-cultural issues in clinical practice. The Liaison Committee on Medical Education of the United States added a standard for medical school education programs to include cultural competency education within their curricula. ${ }^{7}$ While this would need rethinking of teaching-learning activities, it is possible to seamlessly integrate cultural competence training into medical education at different levels. ${ }^{8}$ Such training should include methods for eliciting patients' understanding of illness and their condition. ${ }^{1}$ Exploring patients' beliefs would provide the physician an opportunity of educating the patient while avoiding costly investigations and medicines. Interactive, casebased discussion that highlight cultural issues related to patient management would be an ideal method for teaching cultural competence.

In a bid to minimise disparities in access to and delivery of healthcare, many Western countries have implemented cultural competence in healthcare facilities. ${ }^{9}$ While culturally competent physicians will by no means eliminate disparities in healthcare, it will provide the physician with skills needed to provide highquality holistic care to multicultural societies.

\section{References}

1. Betancourt JR. Cultural competence-marginal or mainstream movement. New England Journal of Medicine 2004; 351; 10 https://doi.org/10.1056/nejmp048033

2. Chandratilake M, Jayarathne YGSW, Karunarathne D. Cultural beliefs and practices of Sri Lankan public as experienced by clinicians. $130^{\text {th }}$ Anniversary International Medical Congress, SLMA 2017; 62(Supplement 1): 40.

3. Betancourt JR, Green AR, Carrillo JE, Owusu AnanehFirempong II. Defining cultural competence: a practical framework for addressing racial/ethnic disparities in healthcare. Public Health Reports 2003; 118: 293-302. https://doi.org/10.1016/S0033-3549(04)50253-4

4. Cross TL, Bazron BJ, Dennis KW, Isaacs MR. Towards a Culturally Competent System of Care: A Monograph on 
Effective Services for Minority Children Who Are Severely Emotionally Disturbed. Georgetown University Child Development Center, CASSP Technical Assistance Center. 1989 https://files.eric.ed.gov/fulltext/ED330171.pdf

5. Like RCL, Barrett TJ, Moon J (Summer 2008). Educating Physicians to Provide Culturally Competent, Patient-Centered Care. Perspectives: A View of Family Medicine in New Jersey 2008; 7(2): 10-20 http://www.ncsl.org/documents/ health/NJAFPHandout52013.pdf

6. Senanayake P, De Silva B, Madumanthi A, Madushani N, Wijerathne, K, Sivayogan S, Wanigatunge, CA. Health Care Related Behavior among Residents of A Selected Medical Officer of Health Area. $2^{\text {nd }}$ International Conference on Multidisciplinary Approaches (iCMA) University of
Sri Jayewardenepura, Sri Lanka. September 2015. http://dr.lib.sjp.ac.Ik/handle/123456789/3929

7. Association of American Medical Colleges (AAMC). Cultural competence education. https://www.aamc.org/media/ 20856/download Accessed April 17th 2021

8. Mainous AG, Xie Z, Yadav S, Williams M, Blue AV, Hong Y. Physician Cultural Competency Training and Impact on Behavior: Evidence From the 2016 National Ambulatory Medical Care Survey. Fam Med. 2020; 52(8): 562-9. https://doi.org/10.22454/FamMed.2020.163135.

9. Handtke O, Schilgen B, Mösko M. Culturally competent healthcare-A scoping review of strategies implemented in healthcare organizations and a model of culturally competent healthcare provision. PLoS ONE 2019; 14(7): e0219971. https://doi.org/10.1371/journal. pone.0219971 